\title{
A metabolite of baicalin detected in rabbit plasma using electrospray mass spectrometry after baicalin administration
}

\author{
Xi-gang Liu MSc, Jin-Zhi Wang, Hong-Juan Shi, Jin-hua Chang MSc, \\ Zhong-si Li MSc, Pei Liu MSc, Cui-zhe Liu PhD
}

\begin{abstract}
X-g Liu, J-Z Wang, H-J Shi, et al. A metabolite of baicalin detected in rabbit plasma using electrospray mass spectrometry after baicalin administration. Curr Res Integr Med 2015;1(X):11-13.

Scutellaria baicalensis Georgi (Huangqin or Chinese Skullcap) has been used to treat inflammatory-related disorders in China and other Asian countries for centuries, and is a major ingredient of many traditional Chinese medicines. The main constituents of the root of Scutellaria, baicalin along with its aglycone, baicalein, are important components of some modern
\end{abstract}

The root of Scutellaria baicalensis Georgi (Huangqin in Chinese) 1 is one of the most widely used traditional Chinese medicines (TCMs), and is officially listed in the Chinese pharmacopoeia (1). It has been used for dissipating heat, moistening aridity, purging fire, detoxifying toxicosis and anti-inflammation (2) expressed in the terms of TCM, as well as anti-cancer (3), anti-bacteria and anti-virus, antioxidant activities (4), reducing total cholesterol levels and decreasing blood pressure in the terms of modern medicine $(5,6)$. Baicalin (baicalein 7-O- $\beta$-glucopyranuronoside [Figure 1]) is a well-known bioactive component in the roots of $S$ baicalensi. Baicalin can protect the myocardium from reperfusion-induced damage in isolated rat hearts via the antioxidant and paracrine (7), inhibit 3T3-L1 preadipocytes differentiation by down-regulation of PDK1/Akt phosphorylation (8), and attenuates brain edema in a rat model of intracerebral hemorrhage (9). Glucuronidation is the main metabolic pathway for baicalin $(10,11)$. Five biliary metabolites have been identified after oral administration of baicalin to rats. The major same metabolites of baicalin in Scutellaria extract and Shuanghuanglian were baicalin6-O- $\beta$ - glucopyranuronoside-7-O- $\beta$-glucopyranuronoside, baicalin6-O-glucoside-7-O- $\beta$-glucopyranuronoside and 6-O-methyl-baicalin -7-O- $\beta$-glucopyranuronoside, baicalin-6-O- $\beta$-glucopyranuronoside and baicalein (11). Three biliary metabolites have been identified after oral administration of baicalin to human. The major same metabolites of baicalin were baicalein-6-O- $\beta$-glucopyranuronoside $\beta 6-O-$ methy- 1 baicalin-7-O- $\beta$-glucopyranuronoside and baicalin (12). Baicalein-6O- $\beta$-glucopyranuronoside has been detected as a major metabolite besides baicalin in plasma after oral administration of baicalin to rats (13). The conjugated metabolites are readily hydrolyzed by the enzyme $\beta$-glucuronidase/sulfatase to free aglycone, and baicalin has been found to be absorbed from the gastrointestinal tract as its aglycone, which is restored to the baicalin parent drug by glucuronidation in the intestine and the liver in vivo (14). The present study investigated metabolites of baicalin in rabbit plasma.

\section{METHODS}

1) Chemicals and reagents: Baicalin (Batch No.111432-200807) and baicalein (Batch No.111595-200604) were purchased from the National Institute for the Control of Pharmaceutical and Biological formulations. In bioavailability studies investigating baicalin, the authors found a substance, as a metabolite of baicalin in rabbit plasma, after oral administration of baicalin. Orally administered pure baicalin and the metabolite was detected in rabbit plasma using high-performance liquid chromatography, and the structure of the metabolite was elucidated using electrospray ionization mass spectrometry methods.

Key Words: Baicalin; HPLC ESI-MSn; Metabolite; Scutellaria baicalensis Georgi

Products (Beijing, China). Methanol (HPLC grade) was purchased from MREDA (USA). Pure water was prepared by distillation of deionized water.

2) Animals: Five male rabbits (mean $[ \pm \mathrm{SD}]$ weight $1.5 \pm 0.2 \mathrm{~kg}$ ) were housed one per cage with water and a solid diet freely available, and maintained at $22 \pm 3^{\circ} \mathrm{C}$ with $40 \%$ to $70 \%$ relative humidity. The rabbits were fasted overnight for at least $12 \mathrm{~h}$ with ad libitum access to water. Each rabbit was given an oral dose of $240 \mathrm{mg} / \mathrm{kg}$ of baicalin. Blood samples were collected from auris edge vein of the rabbit at $1 \mathrm{~h}$ before dosing for blank control and at $1.5 \mathrm{~h}$ after dosing. The blood samples were centrifuged immediately to recover plasma. In all cases, animal experiments scrupulously respected the Department of Health Principles of Using Laboratory Animals.

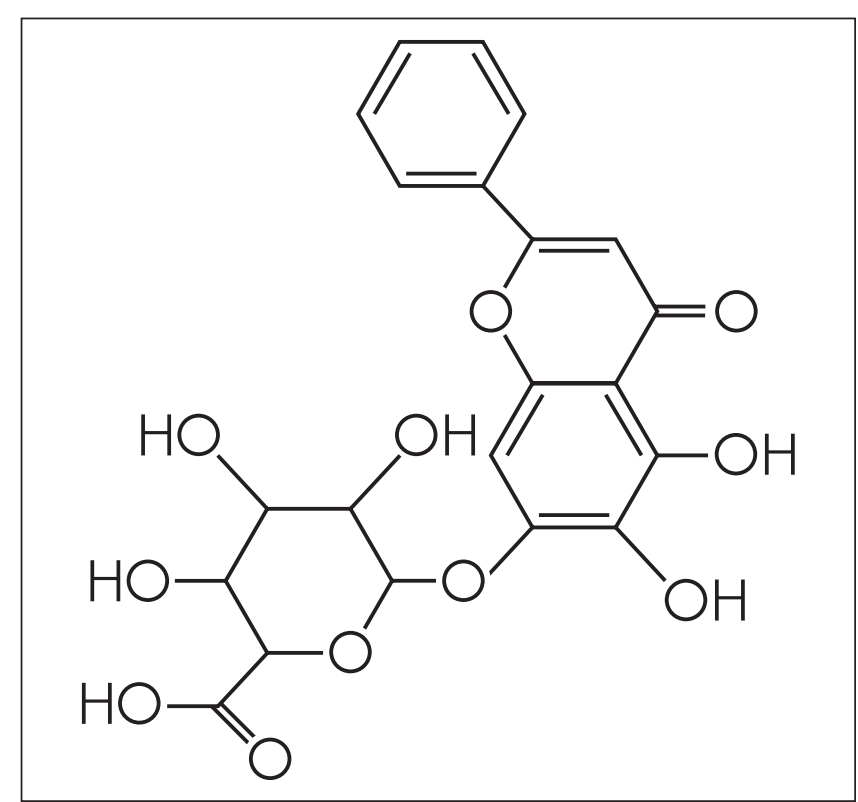

Figure 1) Structure of baicalin

Hebei Province Key Laboratory of Research and Development for Chinese Medicine, Chengde Medical College, Chengde, China

Correspondence: Prof Liu Cui-zhe, Hebei Province Key Laboratory of Research and Development for Chinese Medicine, Chengde Medical College,

Chengde 067000, China. Telephone 86-3142290359, e-mail liucuizhexy@163.com 


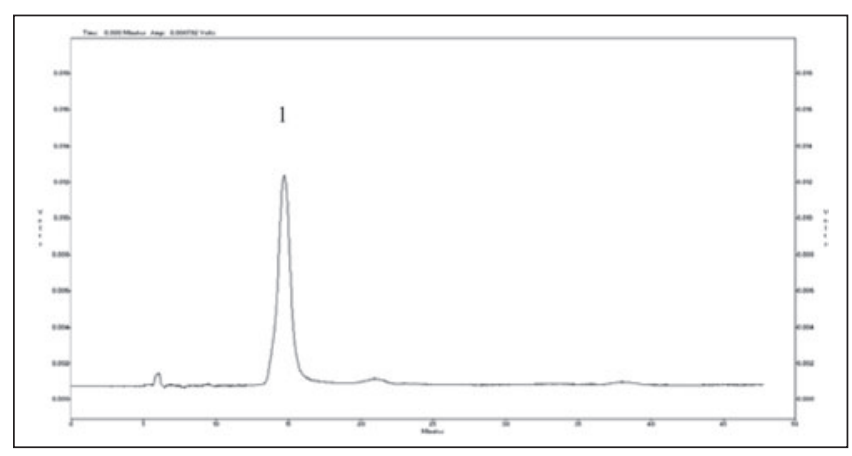

Figure 2) HPLC chromatogram of baicalin (1)

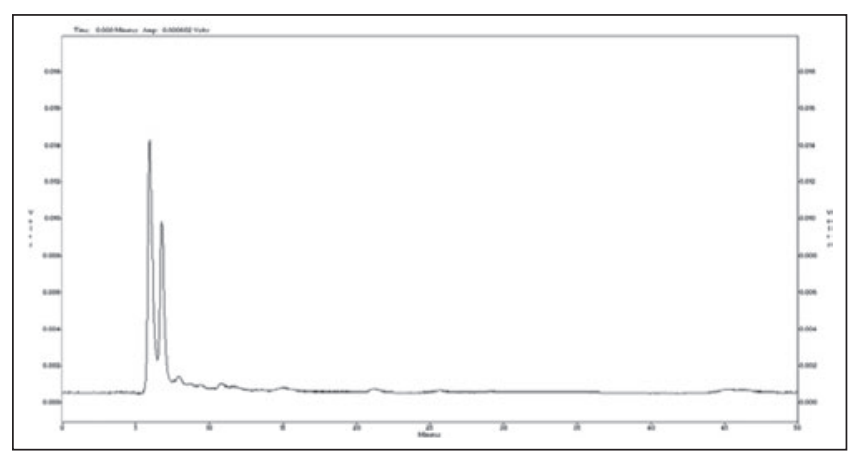

Figure 3) HPLC chromatogram of the blank plasma sample

3) Sample preparation: Blood samples collected from the auris edge vein of the rabbit were immediately centrifuged at $3000 \mathrm{rpm}$ (LG16-W centrifuge, (Beijing, China). The rabbit plasma $(0.2 \mathrm{~mL})$ was mixed with $1.8 \mathrm{~mL}$ of methanol, and the mixture of plasma and methanol was swirled using ZH-2 Automatism Whirlpool Mix Apparatus (Tianjin, China). After sonication of the mixture for $10 \mathrm{~min}$ using CX-500 ultrasonic apparatus (Beijing, China), the mixture was centrifuged again and the supernatant was filtered through a membrane filter $(0.45 \mu \mathrm{m}$, Millipore) and $10 \mu \mathrm{L}$ of filtrate was injected for HPLC analysis.

Baicalin solution (solution A) was prepared by weighting exactly $4 \mathrm{mg}$ of baicalin standard and dissolved into $50 \mathrm{~mL}$ of methanol and diluted, again yielding a $4 \mu \mathrm{g} / \mathrm{mL}$ baicalin methanol solution. At the same time, the following samples for HPLC analysis were prepared by the methods mentioned above. Solution B was a blank plasma sample; solution D was the baicalein standard; solution $\mathrm{C}$ was the plasma sample after oral administration of baicalin to the animals; solution $\mathrm{E}$ was a mixture of solutions $\mathrm{A}, \mathrm{C}$ and $\mathrm{D}$.

4) HPLC analysis: The analysis was performed using a JASCO LC-10A series (Japan) liquid chromatograph equipped with a JASCO pump (model PU-1580), a JASCO detector (model UV-1575) and a column oven. A SUPELCO C18 reverse-phase column $(250 \times 4.6 \mathrm{~mm}$, $5 \mu \mathrm{m}$, Discovery, USA) protected by a guard column was used. A $\mathrm{MeOH}-\mathrm{H}_{2} \mathrm{O}$-formic acid (55:45:0.1, V:V:V) was used as mobile phase at a flow rate of $0.5 \mathrm{~mL} / \mathrm{min}$. Samples were filtered through a membrane $(0.45 \mu \mathrm{m}$, Millipore $)$ and $10 \mu \mathrm{L}$ was injected. Chromatograms were recorded at $279 \mathrm{~nm}$ using the UV detector. The chromatograms of the samples are presented in Figures 2 to 6.

5) LC-MSn analysis: LC-MSn analyses were performed using the Agilent Technologies 6310 Lon Trap LC/MS (USA), Ultra equipped with an electrospray ion source. The separation was performed using a SUPELCO C18 reverse-phase column $(250 \times 4.6 \mathrm{~mm}, 5 \mu \mathrm{m}$, Discovery, USA) with a guard column. $\mathrm{MeOH}-\mathrm{H}_{2} \mathrm{O}$ - formic acid (55:45:0.1, $\mathrm{V}: \mathrm{V}: \mathrm{V})$ was used as mobile phase, at a flow rate of $0.2 \mathrm{~mL} / \mathrm{min}$. Samples were filtered $(0.45 \mu \mathrm{m}$, Millipore $)$ and $50 \mu \mathrm{L}$ was directly injected.

The mass spectral analysis was performed in a positive electrospray ionization mode. The capillary voltages was set at $4.5 \mathrm{kV}$. The nebulizer

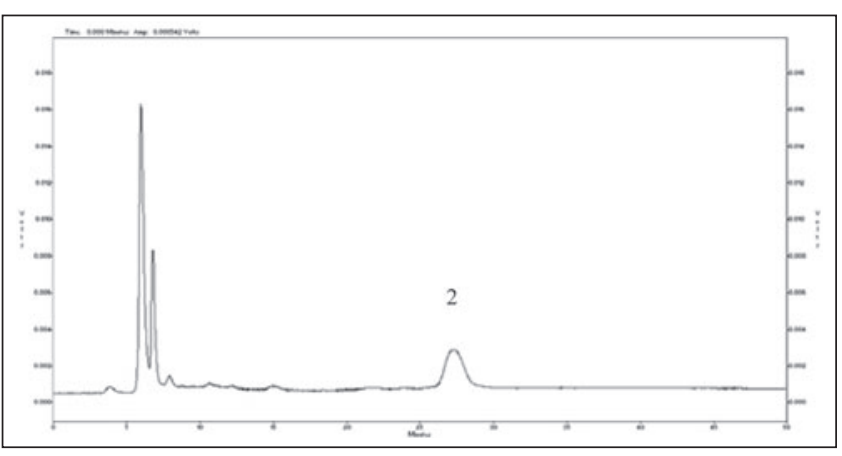

Figure 4) HPLC chromatogram of rabbit plasma sample after administered baicalin. The new metabolite is represented as peak 2

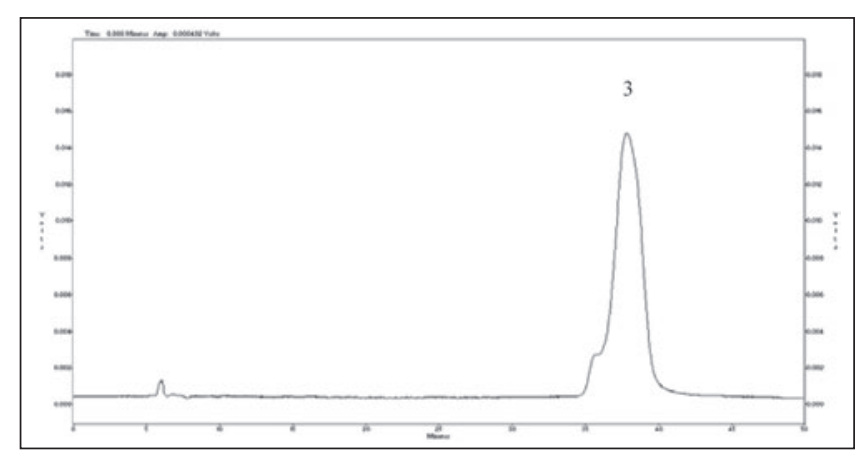

Figure 5) HPLC chromatogram of baicalein. 3 Baicalein

gas was set at $25 \mathrm{psi}$; the vapourizer temperature was set at $350^{\circ} \mathrm{C}$. Collision-induced dissociation (CID) studies were performed using a collision energy of $30 \mathrm{eV}$. The ionspray interface and mass spectrometric parameters were optimized to obtain maximum sensitivity at unit resolution. The LC-MSn spectra of the metabolite of baicalin are presented in Figure 7.

\section{RESULTS AND DISCUSSION}

\section{HPLC identification of baicalin metabolites}

Figure 2 is HPLC chromatogram of baicalin and Figure 3 shows the HPLC chromatogram of the blank plasma sample. Figure 4 is the HPLC chromatogram of rabbit plasma samples after oral administration of baicalin, Figure 5 is HPLC chromatogram of baicalein. Figure 6 shows that the detected peaks in plasma sample after oral administration of baicalin in rabbit was not baicalin and baicalein.

\section{LC-MS/MS identification of the metabolites of baicalin}

As illustrated in Figure 7, the positive electrospray mass spectrum of metabolite of baicalin showed a $(\mathrm{M}+\mathrm{H})+$ ion at $\mathrm{m} / \mathrm{z} 447$. The CID spectrum of $\mathrm{m} / \mathrm{z} 447$ generated only one of fragment ions at $271(\mathrm{M}+\mathrm{H})+$. The fragment ion at 271 was generated from the loss of $176(\mathrm{C} 6 \mathrm{O} 6 \mathrm{H} 8)$ from the ion at $\mathrm{m} / \mathrm{z} 447$. The maximum ultraviolet absorption peak for metabolite of baicalin was $277 \mathrm{~nm}$ and $317 \mathrm{~nm}$, which was different from that of baicalin in $270 \mathrm{~nm}$ and $316 \mathrm{~nm}$. The fragmentation pathway is illustrated in Figure 8. The major metabolites of baicalin was baicalein-6-O- $\beta$-glucopyranuronoside.

Rabbits and rats are different species. Baicalin metabolic components may be different in their plasma, in the present experiment, only one type of metabolic component of baicalin was found, and the content was high, the remainder of the components (including baicalin) were not detected. The reason may be that all baicalin converted into the metabolic component.

In the present study, we found that baicalin was converted mostly into its metabolic component after it was absorbed into the bloodstream of the rabbits. It suggests that it is the metabolic component of baicalin, not baicalin itself, should be measured in the study of baicalin 


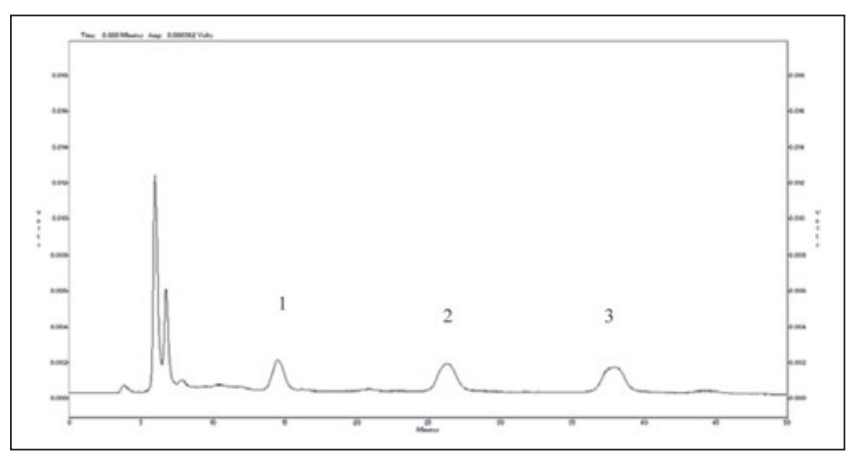

Figure 6) HPLC chromatogram of a mixture of solutions A and C. 1 Baicalin; 2 New metabolite; 3 Baicalein

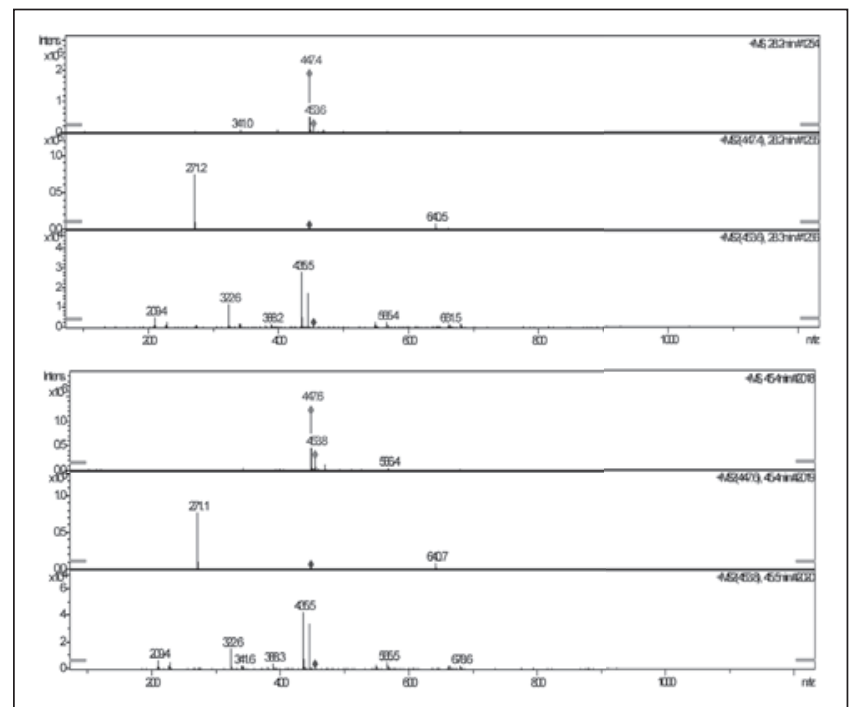

Figure 7) LC-MSn spectra of metabolite of baicalin ( $\mathrm{m} / \mathrm{z} 447)$ obtained using positive ion electrosprary and CID (top: baicalin, bottom: metabolite)

pharmacokinetics and when the drug concentration-time curve was drawn in rabbit .

In our study investigating the relative bioavailability characterisitics of Sanhuang tablets, a new metabolite of baicalin was detected by HPLC. To confirm the finding, pure baicalin was administered orally to rabbits and the same metabolite was detected in the rabbit plasma with HPLC-UV detection. To further elucidate the structure of the metabolite, an HPLC-MSn method was used to analyze the structure. It is a metabolite of baicalin, which is not stable (15). In the present study, we found the metabolite of baicalin was baicalein- $6-O-\beta$-glucopyranuronoside. It was different from the metabolite of baicalin in Sanhuang tablets in our previous study.

ACKNOWLEDGEMENTS: This work was financially supported by the National Natural Science Foundation of China (No. 81073146, 81341143), and the key subject construction project of hebei provincial college; and the Natural Science Foundation of Hebei Province(No.H2014406036). Science and Technology Research Key Project of Higher School in Hebei Province (No.ZH2012050); Science and Technology Research Youth Fund Project of Higher School in Hebei Province (No. 2011133).

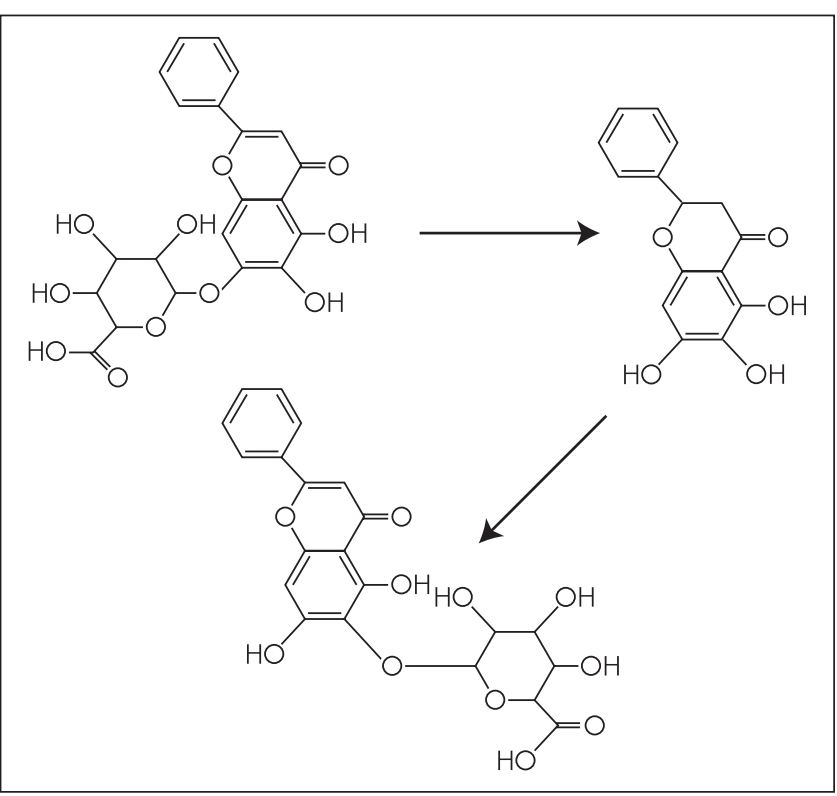

Figure 8) The major metabolic pathway of baicalin in rabbits

\section{REFERENCES}

1. National pharmacopoeia committee. Pharmacopoeia of the People's Republic of China, vol. 1, Chinese medical science and technology Press (Beijing) 2010;282-3.

2. Jung Myung-Ah, Jang Se-Eun, Hong Sung-Woon, et al. The role of intestinal microflora in anti-inflammatory effect of baicalin in mice. Biomol Ther (Seoul) 2012;20:36-42.

3. Shu Yi-Jun, Bao Run-Fa, Wu Xiang-Song, et al. Baicalin induces apoptosis of gallbladder carcinoma cells in vitro via a mitochondrialmediated pathway and suppresses tumor growth in vivo. Anti-cancer Agents Med Chem 2014,14:1136-45.

4. Liu Peng-Fei, Han Fu-Gen, Duan Bin-Bin, et al. Purification and antioxidant activities of baicalin isolated from the root of huangqin (Scutellaria baicalensis gcorsi). J Food Sci Technol 2013;50:615-9.

5. Wang Hongling, Liu Daquan. Baicalin inhibits high-mobility group box 1 release and improves survival in experimental sepsis. Shock 2014,41:324-30.

6. Xin Wen-yu, Song Jun-ke, HE Guo-rong, et al. Progress in pharmacological study and the underlying mechanism of baicalein and baicalin. Chin J New Drugs 2013;22:647-53.

7. Kong Feng, Luan Yun, Zhang Zhao-hua, et al. Baicalin protects the myocardium from reperfusion-induced damage in isolated rat hearts via the antioxidant and paracrine effect. Exp Ther Med 2014;7:254-9.

8. Kwak Dong Hoon, Lee Ji-Hye, Song Kwang Hoon, et al. Inhibitory effects of baicalin in the early stage of 3T3-L1 preadipocytes differentiation by down-regulation of PDK1/Akt phosphorylation. Mol Cell Biochem 2014;385:257-64.

9. Zhou Qing-Bo, Jin Yun-Ling, Jia Qing, et al. Baicalin attenuates brain edema in a rat model of intracerebral hemorrhage. Inflammation 2014;37:107-15.

10. Ki Abe, Inoue O, Yumioka E. Biliary excretion of metabolites of baicalin and baicalein in rats. Chem Pharm Bul 1990;38:209-11.

11. Di Bin, Feng Nian-ping, Liu Wen-ying. Study on metabolism differences of baicalin in compound Shuanghuanglian and Huangqin extract in rats. Chin Pharm J 2005;39:127-30.

12. CHE Qing-ming, HUANG Xin-li, LI Yan-mei, et al. Studies on Metabolites of Baicalin in Human Urine. China Journal of Chinese Materia Medica, 2001,26(11):768-769.

13. Akao Teruaki, Sato Keisuke, He Ju-Xiu, et al. Baicalein 6-O- $\beta$-Dglucopyranuronoside is a main metabolite in the plasma after oral administration of baicalin, a flavone glucuronide of scutellariae radix, to rats. Biol Pharm Bull 2013;36:748-53.

14. Akao T, Kawabata K, Yanagisawa E,et al. Baicalin, the predominant flavone glucuronide of scutellariae radix, is absorbed from the rat gastrointestinal tract as the aglycone and restored to its original form. J Pharm Pharmacol 2000;52:1563-8.

15. Liu cui-zhe. The preliminary study on Sanhuang dispersible tablets and in vivo metabolite of baicalin in Sanhuang dispersible tablets in rabbit. Shenyang Pharmaceutical University Masters Dissertation, 2000.06. 